\title{
Should physician-assisted suicide be accepted in the practice of medicine? A literature review
}

\author{
Author: Yuen W Liao
}

\begin{abstract}
Aims
With life expectancy increasing due to the many advancements in modern medicine, the prevalence of chronic illnesses has also increased, hence some have argued for the need for physicianassisted suicide (PAS) to release these patients from their suffering.

PAS has been a long-standing point of contention within the medical world and has only recently been legalised in a handful of European countries and American states. This review aims to further understand the various legislation set out by these countries and states which have legalised PAS. The studies published on PAS from these countries and states will also be analysed.

This literature review also aims to investigate the intricacies of PAS by considering both arguments for and against PAS. All arguments will be analysed unbiasedly to formulate a better understanding of PAS.
\end{abstract}

\section{Methods}

A literature search was conducted using the online databases (PubMed and Philpapers) for the selection of papers relevant to the aims of this review.

To optimise the quality of findings, a pre-determined set of inclusion and exclusion criteria was applied. A paper was deemed significant if it was about the ethical, moral or professional dilemmas surrounding PAS; PAS laws and safeguards; and arguments for or against PAS.

\section{Results}

A total of 21 papers were included in the final analysis. From analysing all the papers, four common themes emerged hence this review focused on these themes.

These themes were identified and extracted from the papers to be critically analysed and discussed. The four common themes found were respect for autonomy, protecting the vulnerable, right to die and the role of the doctor in PAS. With each theme, we aimed to provide arguments from both sides and also backed them up with relevant statistics and data. It was not beyond this review to discount the importance of patient's views on this sensitive subject, hence both quantitative and qualitative studies were included in this review. The statistics on PAS were obtained from audits conducted in the various countries and states which have legalised PAS.

Concepts surrounding mental capacity, national legislations, safeguards and the slippery slope argument were also considered in the discussion.

\section{Conclusion}

PAS is a real, multifaceted and complicated bioethical issue. A perfect balance between relieving suffering and respecting life has to be struck to ensure the safety of all parties involved. Healthy discourse and debate must take place to ensure this balance is found.

With both sides of the argument considered, it is difficult to determine which camp has the better argument as both raised valid points which must be considered seriously. The question of whether PAS deserves a place in modern medicine has proven to be a complicated and intricate one and there is no doubt that the debate will continue.

In conclusion, the findings in this review suggests that PAS has a place in modern medicine however it is impossible at this point of time to determine to what extent the influence of PAS should go.

\section{Conflict of interest statement}

None declared. 